\title{
Development of polyethersulfone (PES)/reduced graphene oxide nanocomposite nanofiltration membrane
}

\author{
Norzakiah Hamzah a, Fasihah Johary ${ }^{a}$, Rosiah Rohani ${ }^{b}$, Syazrin Syima Sharifuddin ${ }^{c}$, Mohd \\ Hafez Mohd Isa a, \\ Faculty of Science and Technology, Universiti Sains Islam Malaysia, Bandar Baru Nilai, 71800 Nilai, Negeri Sembilan, Malaysia. \\ ${ }^{b}$ Faculty of Engineering and Built Environment, Universiti Kebangsaan Malaysia, 43600 UKM Bangi, Selangor, Malaysia. \\ c National Hydraulic Research Institute of Malaysia, Jalan Putra Permai, 43300 Seri Kembangan, Selangor, Malaysia. \\ * Corresponding author: m.hafez@usim.edu.my
}

\section{Article history}

Received 9 May 2019

Revised 2 July 2019

Accepted 5 January 2020

Published Online 16 August 2020

\begin{abstract}
Polyethersulfone (PES) is a polymeric material that is commonly used due to its chemical resistance, high mechanical strength, and thermal stability. The improvement of PES hydrophilicity and anti-fouling properties can be facilitated by mixing additives into the polymer casting solution. This study involved the preparation and characterization of PES/pluronic F108/chitosan/reduced graphene oxide (rGO) nanocomposite nanofiltration membrane. The modified PES membrane was developed by employing the phase inversion method via immersion precipitation. The effect of varying concentration of rGO nanocomposite ranging from 0.05 to $0.20 \mathrm{wt} \%$ in the fabricated membrane was evaluated in terms of membrane pure water permeation (PWP), salt rejection, morphology, and hydrophilicity. Images obtained from scanning electron microscopy (SEM) showed increasing rGO nanocomposite concentration in the casting solution, resulted in the increase in membrane sub layer thickness, hydrophilicity, salt rejection, and improvement in the membrane pore structure. Fourier transforminfrared (FTIR) spectroscopy confirmed the presence of the hydrophilic functional group and chemical compound in the prepared membrane. PES with $0.20 \mathrm{wt} \%$ of $\mathrm{rGO}$ nanocomposite obtained the highes PWP at $28.64\left(\mathrm{~L} / \mathrm{m}^{2} \mathrm{~h}\right)$ at 5 bar pressure, whereas the highest rejections of monovalent and multivalent salt were obtained by PES with $0.15 \mathrm{wt} \% \mathrm{rGO}$ nanocomposite with $75 \%$ and $89 \%$ rejections, respectively at 5 bar pressure. This study shows that the utilization of rGO nanocomposite as an additive in the PES casting solution can enhance nanofiltration membrane performance and morphological structure.
\end{abstract}

Keywords: nanofiltration membrane; reduced graphene oxide; salt rejection

\section{INTRODUCTION}

Membrane technology has been explored extensively to find ways to enhance their function and application in various industries. According to Fang et al. (2015), water flux and selectivity of porous polymeric membranes highly dependent on their structure. However, the main problem in handling membrane technology and applications lies in fouling. Fouling is an inevitable phenomenon even with pretreatment, optimum system design, and excellent anti-fouling properties. Membrane fouling causes flux decline, thus reducing the efficiency and economic benefits of the membrane processes (Wahab et al., 2011).

Polyethersulfone (PES) is a polymeric material that is widely utilized in separation fields (Hosseini, 2016). PES is used due to its chemical resistance, high mechanical strength, and thermal stability. However, the disadvantage of PES includes hydrophobicity, which can cause membrane fouling. The improvement of PES hydrophilicity and anti-fouling properties can be facilitated by mixing additives in the polymer casting solution (Liu et al., 2003).

The functional groups of reduced graphene oxide (rGO) enable faster water permeation due to the interaction between the functional group and ions or water molecule (Pei et al., 2016). Meanwhile, Pluronic F108 displays great surface activity and micellization in water. This characteristic of pluronic can potentially change the surface properties and pore structure of membranes and, thus, result in high flux and anti-fouling properties. Therefore, the fabrication of PES/pluronic F108/chitosan/rGO nanofiltration membranes was performed to overcome membrane fouling and increasing hydrophilicity of PES membrane.

\section{EXPERIMENTAL}

\section{Materials}

Chemicals, such as N-methyl-2-pyrrolidone (NMP), were used to dissolve the polymeric membrane material of PES. Chitosan and rGO nanocomposite act as additives, whereas pluronic F108 is the surfactant in the dope solution. Ethanol and n-hexane were utilized to improve the structure of the membrane as a pre-treatment condition. All of the chemicals and solvents were purchased from either Sigma Aldrich or MERCK.

\section{Dope Preparation and Membrane Casting}

The membranes were fabricated using the phase inversion technique. The mixture of NMP solvent and deionized water was stirred at $30-50^{\circ} \mathrm{C}$, while dried PES was added gradually to the round bottom flask until a homogenous solution was formed. Pluronic F108, chitosan, and rGO nanocomposite were added to polymeric solution and mixed for six to eight hours to form a homogenous solution. The polymeric 
solution was left to cool at room temperature for 24 hours. The dope compositions used for the fabrication of membrane is tabulated in Table 1.

Table 1 Dope component and formulation (weight percentage, wt \%).

\begin{tabular}{lcccccc}
\hline Dope (M) & PES & NMP & $\mathrm{H}_{2} \mathrm{O}$ & $\begin{array}{l}\text { Chitosan } \\
\text { (additive) }\end{array}$ & $\begin{array}{l}\text { rGO } \\
\text { (additive) }\end{array}$ & $\begin{array}{l}\text { Pluronic } \\
\text { F108 } \\
\text { (surfactant) }\end{array}$ \\
\hline PES/rGO-1 & 21 & $\begin{array}{c}70.9 \\
5\end{array}$ & 4 & 2 & 0.05 & 2 \\
PES/rGO-2 & 21 & $\begin{array}{c}70.9 \\
0\end{array}$ & 4 & 2 & 0.10 & 2 \\
PES/rGO-3 & 21 & $\begin{array}{c}70.8 \\
5\end{array}$ & 4 & 2 & 0.15 & 2 \\
PES/rGO-4 & 21 & $\begin{array}{c}70.8 \\
0\end{array}$ & 4 & 2 & 0.20 & 2 \\
& & & & & & \\
\hline
\end{tabular}

The next process was the casting, which was carried out by pouring the casting solution on the glass plate. The membrane was casted using a casting knife with a fixed casting time of about 10 seconds. The glass plate was immersed in a coagulation bath with a temperature of $25^{\circ} \mathrm{C}$. In the coagulation bath, phase separation occurred where the exchange between solvent and non-solvent was involved. The membrane was peeled off manually from the glass plate and transferred to separated coagulation bath for the complete removal of any solvent (Qadir et al., 2016). Subsequently, the membranes were immersed in ethanol solution for 24 hours, followed by n-hexene immersion for 3 hours. The membrane was then transferred and dried at room temperature for 24 hours.

\section{Membrane performance and characterization evaluation}

Pure water permeation (PWP) is a method used to determine the pure water flux of each fabricated membrane by using a dead-end filtration cell. In this study, the membrane samples were pressurized with deionized water at varied pressure range from 3 to 5 bar. The permeation of water flux was determined by measuring the water passing through the membrane at a rate $\mathrm{L} / \mathrm{m}^{2} \mathrm{~h}$, applying equation (1), where $\mathrm{Q}$ is the permeate volume (L), $\mathrm{A}$ is the membrane's active surface area $\left(\mathrm{m}^{2}\right)$, and $\mathrm{T}$ is the time taken for the permeation process (s).

On the other hand, the salt rejection test was evaluated using 0.01 $\mathrm{M}$ of $\mathrm{NaCl}, \mathrm{MgCl}_{2}, \mathrm{Na}_{2} \mathrm{SO}_{4}$, and $\mathrm{MgSO}_{4}$ solutions, respectively, at 5 bar employing a dead-end filtration cell. The solute rejection from permeate was collected and measured according to the conductivity meter, whereas the flux rate of salt rejection was measured by equation (2). Equation (2) was used to calculate the retentate, where $\mathrm{Cp}$ and $\mathrm{Cf}$ are the solute concentration of permeate and feed respectively.

Pure water permeation $(\mathrm{Jw})=\frac{Q}{A \Delta T}$

Retention of rejection $(\mathrm{f} \%)=\left(1-\frac{C_{p}}{C_{f}}\right) \times 100 \%$

The nanofiltration membranes were characterized using scanning electron microscopy (SEM) and Fourier transform-infrared (FTIR) spectroscopy. The objective of utilizing the SEM was for the visual observation of membrane morphological structure, while FTIR spectroscopy was used to determine the functional group and chemical composition of the membranes. The prepared membrane was mounted in a position in which the skin surface faced the electron beam for the SEM analysis, and all spectra captured for FTIR spectroscopy were recorded in a wavelength range form.

\section{RESULTS AND DISCUSSION}

\section{Pure water permeability}

The varying concentration of rGO nanocomposite in membrane formulation as shown in Table 1 was an important parameter to determine the membrane performance and morphological structure. One way of assessing membrane performance was through measurement of pure water permeability (PWP), as presented in Fig. 1.
PWP increases with the concentration of rGO nanocomposite in the casting solution. In this measurement, PES/rGO-1 membrane obtained the lowest PWP at $1.73\left(\mathrm{~L} / \mathrm{m}^{2} \mathrm{~h}\right)$, at 5 bars. The PWP of the membranes improved gradually to $2.20\left(\mathrm{~L} / \mathrm{m}^{2} \mathrm{~h}\right), 14.12\left(\mathrm{~L} / \mathrm{m}^{2} \mathrm{~h}\right)$, and $28.74\left(\mathrm{~L} / \mathrm{m}^{2} \mathrm{~h}\right)$ at 5 bars for PES/rGO-2, PES/rGO-3, and PES/rGO-4, respectively. This gradual increase in PWP might be due to the increase in pore size with the presence of filler nanoparticles. Moreover, pluronic F108 and chitosan enhances macrovoid presence, resulting in high PWP. According to Susanto and Ulbricht (2009), pluronic F108 possesses higher hydrophilic-lipophilic balance (HLB), which can be correlated to higher hydrophilicity and later resulting in higher membrane permeability. The results show that the addition of rGO nanocomposite has improved membrane PWP, and their optimal weight percentage is an important parameter to achieve a better-performing membrane.

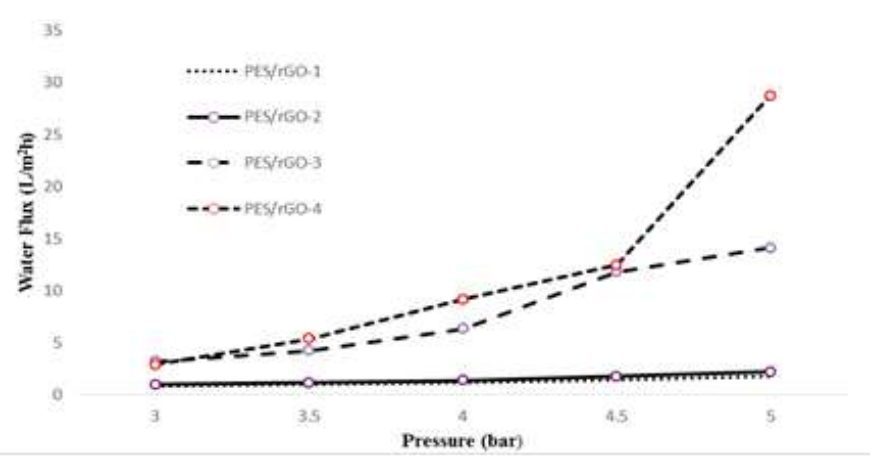

Fig. 1 Pure water permeation performance on different pressure.

\section{Salt Rejection}

The salt rejection test of the fabricated membrane was performed with solution of $\mathrm{NaCl}, \mathrm{MgCl}_{2}, \mathrm{Na}_{2} \mathrm{SO}_{4}$, and $\mathrm{MgSO}_{4}$ at $0.01 \mathrm{M}$ concentration. Each membrane was pressurized at 5 bar, using a deadend filtration cell. The effect of different amount of rGO nanocomposite on the membrane performance in terms of flux and salt rejection is shown in Fig. 2.

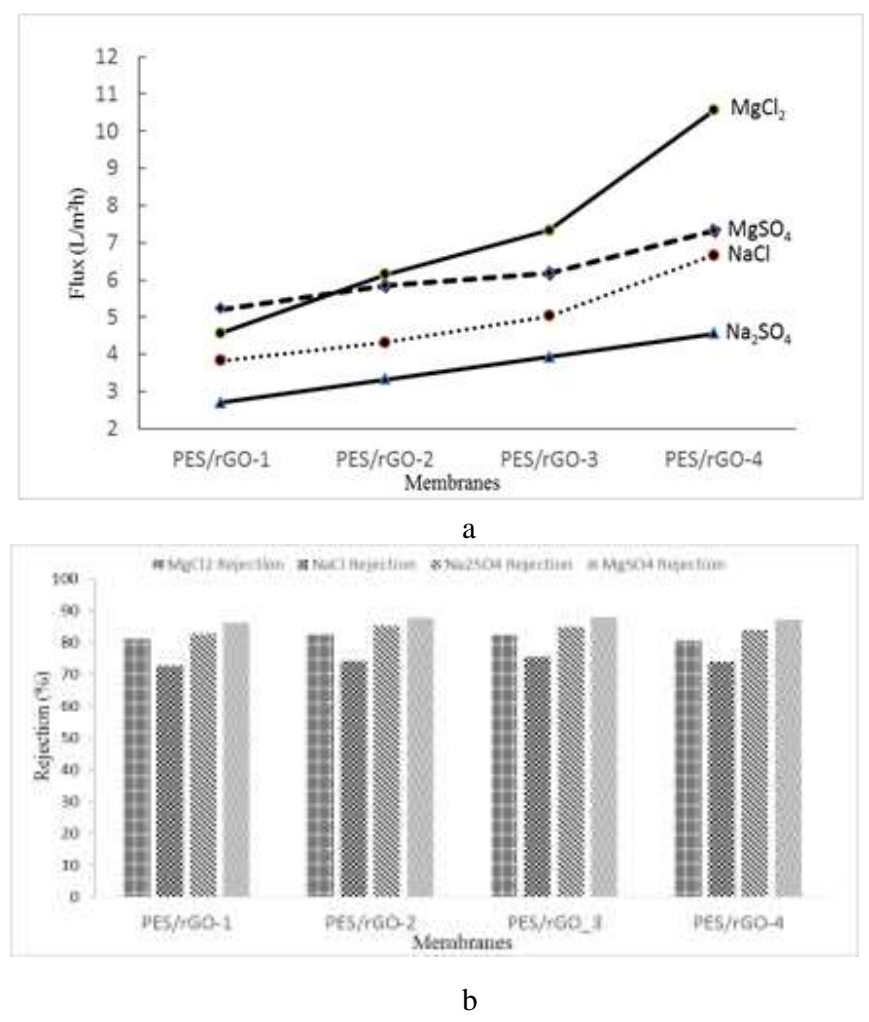

Fig. 2 a) Salt permeation flux; and b) salt rejection test of fabricates membranes. 
Fig. 2 (a) shows permeability of membranes increases with increasing concentration of $\mathrm{rGO}$ nanocomposite in the casting solution. This increasing trend might be due to the increasing membrane hydrophilicity as similarly observed in PWP measurement as presented in Fig. 1. In addition, increasing amount of rGO nanocomposite also causes more formation of finger-like pore structure and larger pore size of membranes. Salt rejection test in Fig. 2 (b) for the four types of membranes were observed to be following the order of: PES/rGO- $1<$ PES/rGO-2 < PES/rGO-4 < PES/rGO-3, ranging from $73 \%$ to $88 \%$ of salts rejection; although the difference of rejection tests performance of the four types of membranes were generally minimal. High rejection of salts was due to the negatively charged hydrophilic functional groups, such as hydroxyl and carboxyl groups on the surface of rGO nanocomposite, which build up high zeta potential through induction of negative charges on the membrane surface. Electrostatic interaction of the negatively charged salt and the membrane surface result in the increase of Donnan repulsion, resulting in an increase in salt rejection (Rezaee et al., 2015). According to Omidvar (2015), size exclusion (steric hindrance), Donnan repulsion, and adsorption on the membrane surface are factors characterizing nanofiltration membrane separation performance and selectivity.

\section{Membrane Characterization}

\section{Fourier-Transform Infrared (FTIR) Spectroscopy}

The presence of various functional group was analyzed using the Varian 300 FTIR spectroscope. Generally, the detected bands indicated the functional groups present in the fabricated membranes. Fig. 3 presents the transmittance bands of each membrane.

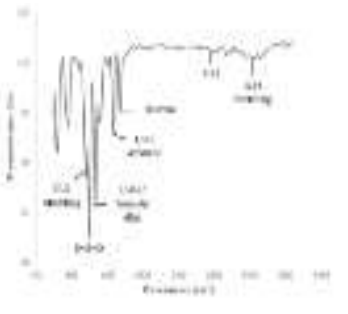

a

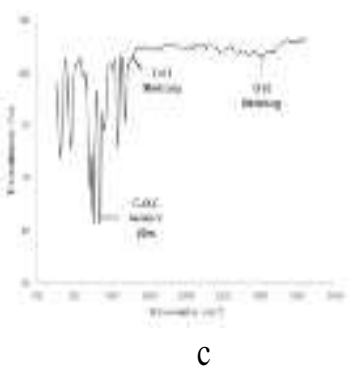

c

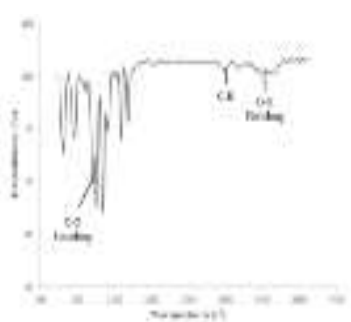

b

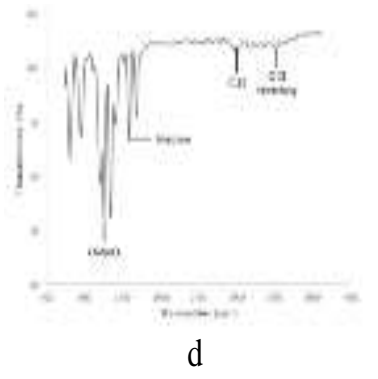

Fig. 3 (a,b,c,d) FTIR transmittance bands showing the molecular activity of the membranes

IR spectra for PES/rGO-1 show that the prominent adsorption peak appeared at $1157 \mathrm{~cm}^{-1}, 1580 \mathrm{~cm}^{-1}$ and $1486 \mathrm{~cm}^{-1}$, which relate to the stretching vibration of $\mathrm{SO}_{2}$, aromatic band from benzene ring, and $\mathrm{C}=\mathrm{C}$ bond stretching respectively. Those peaks indicate the appearance of PES. Meanwhile, the peak $3465 \mathrm{~cm}^{-1}$ reveals the typical rGO nanocomposite characteristic that is assigned to the stretching vibration of $\mathrm{O}-\mathrm{H}$ bond. The band $1712.18 \mathrm{~cm}^{-1}$ can be attributed to the carbonyl stretching vibration $(\mathrm{C}=\mathrm{O})$, and that indicates the presence of a carboxyl functional group. In addition, the adsorption peak at $1152 \mathrm{~cm}$ ${ }^{1}$ can be assigned to the C-O bond stretching vibration of pluronic F108 (Susanto \& Ulbritch, 2009). It can be seen from Figures 3 (a), (b), (c), and (d) that all membranes exhibit similar patterns related to spectrum. The membranes had similar composition with the only difference being in the concentration levels of rGO nanocomposite in the casting solution.

\section{Scanning Electron Microscope (SEM)}

A scanning electron microscope (Hitachi e-1010) was employed to record the images of the microstructure of membrane cross-section. The morphology of PES/rGO-1 and PES/rGO-4 membrane is presented in Fig. 4 (a) and (b) at a magnification of 750x. The cross-section images of the membrane as presented in Fig. 4 show both membranes having sponge-like structure embedded in the area between the micropores. In addition, PES/rGO-4 having wider pores and more sponge-like structures in comparison to PES/rGO-1, with the former having higher amount of rGO nanocomposite in the casting solution.

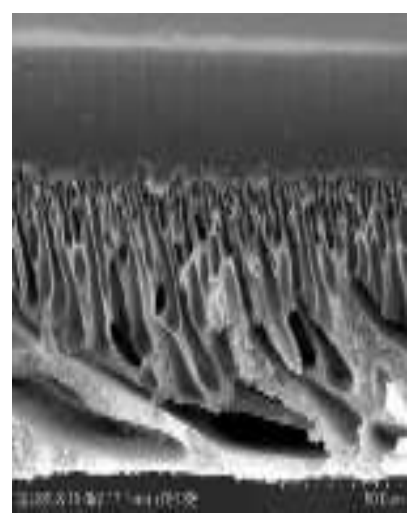

a

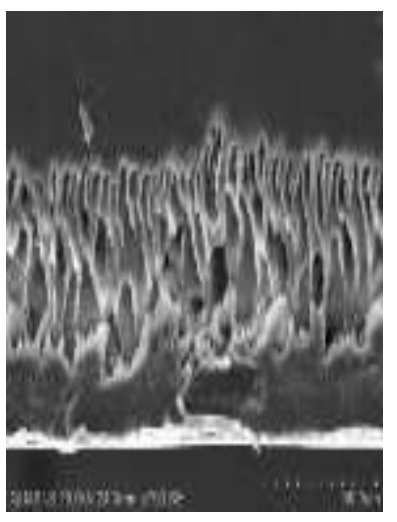

b
Fig. 4 a) SEM images of PES/rGO-1 and b) SEM images of PES/rGO-4 of membranes cross-section

SEM cross-section images in Fig. 4 show both membranes having support layers with similar thicknesses, but with different structures. The support layer of the PES/rGO-4 membrane is more porous than the PES/rGO-1 membrane, which has a cellular structure. According to Moarefian et al. (2014), a porous membrane is influenced by molecular and pore size distribution, with higher pore density and porous sub layer will result to better permeation flux.

\section{CONCLUSION}

In this study, a new formulation of nanofiltration membrane was developed using polyethersulfone (PES) and pluronic F108, chitosan, and reduced graphene oxide ( $\mathrm{rGO}$ ) nanocomposite. The different concentration of rGO nanocomposite resulted to different membrane morphology, porosity, and hydrophilicity. In this study, PES/rGO-4 displayed the highest PWP at $28.74\left(\mathrm{~L} / \mathrm{m}^{2} \mathrm{~h}\right)$, and having larger pore size due to the presence of higher amount of filler nanoparticles in the form of rGO nanocomposite. Moreover, the high salt rejection test was obtained for all membranes in the range of $73 \%$ to $88 \%$. Finally, through the FTIR characterization, it was verified that all functional groups of composition of the membranes are present in the membranes. In conclusion, rGO nanocomposite/chitosan/pluronic F108 are a good combination of membrane dope formulation, as it results in a considerably high permeation flux and high salt rejection.

\section{ACKNOWLEDGEMENT}

This research was supported by the Fundamental Research Grants Scheme, Ministry of Higher Education of Malaysia (FRGS-FST51716-50) and National Hydraulic Research Institute of Malaysia grant (NAHRIM-FST-40816-00). 


\section{REFERENCES}

Wahab, A., Hilal, N., Ying Pei L., Hasyimah, I. N., Raslan, R. 2011. Atomic force microscopy as a tool for assymetric polymeric membrane characterization. Sains Malaysiana 40(3), 237-244.

Fang, X., Li, J., Li, X., Sun, X., Shen, J., Han, W., Wang, L. 2015 Polyethyleneumine an effective additive for polyetehruslfone ultrafiltration membrane with enhance permeability and selectivity. Journal of Membranes Science, 476,216-223.

Hosseini, S. M., Bagheripour, E., Hamidi, A. R., Moghadassi, A. R., Madaeni, S. S. 2016. Fabrication of PES- based nanofiltration membrane modified by composite PAA-co-PMMA-g-ZnA nanoparticles. Journal of Iranian Chemical Society, 13(10), 1749-1758.

Liu, Y., Koops, G. H., Strathmann, H. 2003. Characterization of morphology controlled polyethersulfone hollow fiber membranes by the addition of polyethylene glycol to the dope and bore liquid solution. Journal of Membrane Science, 223(1-2), 187-199.

Moarefian, A., Golestani, H. A., Bahmanpour, H. 2014. Removal of amoxicillin from wastewater by self-made Polyethersulfone membrane using nanofiltration. Journal of Environmental Health Science and Engineering, 12(1), 127.

Omidvar, M., Mohammad, S., Seyed, M. M., Ehsan, M., Hoda, S. 2015. Preparation of hydrophilic nanofiltration membranes for removal of pharmaceuticals from water. Journal of Environmental Health Science and Engineering, 13, 42.

Pei, J., Zhang, X., Huang, L., Jiang, H., Hu, X., 2016. Fabrication of reduced graphene oxide membranes for highly efficient water desalination. Journal Society of Chemistry, 104.

Qadir, D., Mukhtar, H., Keong, L. K. 2016. Synthesis and characterization of polyethersulfone/carbon molecular sieve based mixed matrix membranes for water treatment applications. Procedia Engineering, 148, 588-593.

Rezaee, R., Nasseri S., Mahvi A. H., Nabizadeh R., Mousavi S. A., Rashidi A. Jafari A., Nazmara S. 2015. Fabrication and characterization of a polyethersulfone-graphene oxide nanocomposite membrane for arsenate rejection from water. Journal of Environmental Health Science \& Engineering, 13(61), 1-11.

Susanto, H., Ulbricht, M. (2009). Characteristics, performance and stability of polyethersulfone ultrafiltration membranes prepared by phase separation method using different macromolecular additives. Journal of Membrane Science, 327(1-2), 125-135. 